\title{
Ergonomic Design of Future Production Systems
}

Increasing global competition and the resulting cost pressures call for premium-quality products and highly effective manufacturing processes. Moreover, in the face of short product life cycles and customers' demand for variety, markets demand flexible adaptation to changing conditions. In order to stay competitive, it is crucial for companies in high-wage countries to anticipate customer-specific needs, individualize their processes and react extremely flexibly. Therefore, innovative production systems are required that are able to adapt continuously to changing product structures and the corresponding production and assembly processes [1].

One approach to achieving a higher degree of adaptability is to design flexible automated production systems. The competitive advantage is thus improved, giving manufacturers the ability to allocate functions dynamically and to make human-machine interaction more effective. The Cluster of Excellence "Integrative Production Technology for High-Wage Countries" focuses on this approach and incorporates key technologies by combining expertise from the fields of production engineering, material sciences and human factors to provide science-based solutions. The aim is to develop the fundamentals of a sustainable production strategy as well as to build methods and models that help us understand, predict and control the behaviour of complex, socio-technical production systems [1].

However, especially in high-wage countries, the level of automation in complex, socio-technical production systems has already been taken so far that, according to some kind of "law of diminishing returns", a further increase will not lead to a significant increase in productivity. As a result, the number of process errors is significantly reduced by automation but the severity of the potential consequences of a single error increases disproportionately. Therefore, human decisions and actions when planning, monitoring, and optimizing complex production processes will also be crucial in the future [2]. In fact, increasing technological complexity in future production systems will often require intensified human intervention, especially for ramp-up, supervisory control and reconfiguration. Hence, future production systems should focus on integrating the human operator and his or her extraordinary problem-solving abilities, creativity, and sensorimotor skills.

This special issue on the ergonomic design of future production systems contains five selected contributions that were part of a special session during the 5th International Conference on Applied Human Factors and Ergonomics (AHFE) held in July 2014 in Kraków, Poland. The special issue focuses on innovative methods for designing future production systems and gives the reader a concise overview of recent work in the field.

Kuz, Bützler and Schlick have analysed the research question of how anthropomorphism in the motion behaviour of a gantry robot in self-optimizing assembly systems affects the predictability of target positions during placement tasks. To investigate this question, they developed a simulation consisting of a robotized assembly cell, which they used to conduct a laboratory study. The virtual gantry robot was 
controlled using human placing movement trajectories. The task of the participants was to predict the target of a placing movement as accurately and quickly as possible. Results show that the anthropomorphic velocity profile leads to a significantly shorter prediction time $(\alpha=0.05)$. Moreover, the error rate and the mental effort were also significantly less for the anthropomorphic velocity profile.

Schmidtler, Knott, Hölzel and Bengler analyse and evaluate new technologies like exoskeletons, collaborative robots and orthosis via different methods for ergonomic assessment such as respiratory analysis, motion tracking and force measuring to develop collaborative assistance systems that improve human acceptance and well-being. Results show that implementing the presented concepts for assistance systems could achieve a meaningful reduction in physical and cognitive stress.

With specific regard to computer-aided process planning, "Ergotyping" tools have been developed that permit computer-based ergonomic evaluation using a digital human model. In their paper, Kamusella, Scherstjanoi and Schmauder introduce two tools designed for hazard and risk assessment. Based on the ergonomic parameters of different established methods, the "Body Forces" tool assesses cases of force application that include the arm-shoulder system and whole body system. The "Manual Handling" tool evaluates the risk of health hazards of load handling, referring to the Key Indicator Method for activities involving lifting, holding and carrying issued by the Federal Institute for Occupational Safety and Health. This tool permits evaluation for both homogenous and heterogeneous cases of manual load handling.

Müglich, Sinn-Behrendt, Schaub and Bruder developed a capability database for the prospective design of capability-appropriate work systems. The capability database facilitates evaluation of the tolerability of the work by considering the physical capabilities and respective limitations for a specific exposure time. Furthermore, it presents data on the development of capabilities with increasing age.

The paper by Ziefle, Brauner and Speicher is concerned with the investigation of data presentations and information complexity on usability, taking enterprise resource planning systems as an example. Strategic decisions still depend on having humans in the loop who can perceive, understand and process increasingly complex information. The information complexity (number of lines per table, number of digits, specificity of labels) and operators' cognitive ability (perceptual speed) were examined for decision speed and accuracy. The results show that decision speed decreased with increasing information complexity. In addition, the perceptual speed of operators moderated their performance. Therefore, it can be deduced that an ergonomic information presentation is especially important for human performance in resource planning and to balance cognitive load.

We would like to thank the authors of this special issue for sharing their findings with us, without which this ambitious publication project would not have been possible. The German Research Foundation (DFG) deserves special acknowledgement for its kind support within the Cluster of Excellence "Integrative Production Technology for High-Wage Countries".

Guest Editors

Christopher M. Schlick Jennifer Bützler

Institute of Industrial Engineering and Ergonomics RWTH Aachen University, Germany

\section{References}

[1] C. Brecher et al., Integrative Production Technology for High-Wage Countries. Springer, Berlin, Heidelberg, 2012.

[2] J.D. Lee, Human Factors and Ergonomics in Automation Design, Handbook of Human Factors and Ergonomics. Third Edition, 2006, pp. 1570-1596. 